\title{
CREB and COUP-TF Mediate Transcriptional Activation of the Human Immunodeficiency Virus Type 1 Genome in Jurkat T Cells in Response to Cyclic AMP and Dopamine
}

\author{
Olivier Rohr, Christian Schwartz, Dominique Aunis, and Evelyne Schaeffer* \\ Unité 338 INSERM, 67084 Strasbourg Cedex, France
}

\begin{abstract}
Infection of lymphocytes by the human immunodeficiency virus type 1 (HIV-1) is associated with an increase in intracellular cAMP levels. Recent studies have shown that lymphocytes are able to synthesize and bind the dopamine, known to affect multiple cellular pathways, such as the cAMP pathway. Here we have investigated the molecular mechanisms by which cAMP and dopamine regulate HIV-1 gene transcription in Jurkat T cells. Transient expression experiments revealed that dopamine and forskolin lead to a synergistic stimulation of long terminal repeat (LTR)-driven transcription. This action is mediated through the cAMP response element binding (CREB) protein and chicken ovalbumin upstream promoter transcription factor (COUP-TF). CREB and COUP-TF act indirectly through the minimal $-40 /+80$ and $-68 /+80$ LTR region, respectively. We have previously demonstrated that COUP-TF stimulates HIV-1 transcription via the $-68 /+29$ LTR region without direct DNA binding. Here, gel supershift experiments show that CREB does not directly bind to the $-45 /+85$ proximal LTR sequences. Moreover, our data reveal novel functional interactions between COUP-TF and CREB, which lead to synergistic CAMP- and dopamine-induced transactivation of the HIV-1 LTR. These findings reveal that dopamine-induced signals and the cAMP pathway stimulate HIV-1 gene transcription in lymphocytes by converging to the minimal $-68 /+80$ LTR region, through the transcription factors CREB and COUP-TF. J. Cell. Biochem. 75:404-413, 1999. ๑1999 Wiley-Liss, Inc.
\end{abstract}

Key words: HIV-1; gene regulation; trancription factors; CAMP; dopamine

Infection by the human immunodeficiency virus type 1 (HIV-1) leads to qualitative immune deficiencies which relate to interference with second messengers pathways in infected $\mathrm{T}$ cells. The importance of the cAMP signaling pathway during the course of HIV-1 infection has been well established. Levels of intracellular cyclic AMP (cAMP) are higher in HIVseropositive subjects than in controls [Hofman et al., 1993b]. An increase in intracellular levels of cAMP has been reported in T-cell lines [Nokta and Pollard, 1991] and normal lymphocytes infected with HIV-1 [Hofmann et al., 1993]. Moreover HIV-1 replication is a cAMP-dependent event, since dibutyryl cAMP and forskolin, an activator of adenylate cyclase, enhance viral

Grant sponsor: Institut National de la Santé et de la Recherche Médicale.

*Correspondence to: Evelyne Schaeffer, Unité 338 INSERM, 5 rue Blaise Pascal, 67084 Strasbourg Cedex, France.

E-mail: schaeffer@neurochem.u-strasbg.fr

Received 28 December 1998; Accepted 28 April 1999 replication in MT-4 cells [Nokta and Pollard, 1992]. It is well known that increased intracellular cAMP levels cause stimulation of protein kinase A (PKA), which leads to activation of the CREB/ATF transcription factor family through PKA-mediated phosphorylation. This phosphoCREB then recruits the adaptor CREB-binding protein $(\mathrm{CBP})$ and basal transcription factors, which lead to increased promoter activation [for review see Nordheim, 1994]. A recent report revealed that HIV infection rapidly increases CREB/ATF binding activity and levels of CREB/ATF proteins in a T-cell line infected by HIV-1 [Rabbi et al., 1997].

The neurotransmitter dopamine is present as free, conjugated and intraplatelet free circulating forms in plasma [Marasini et al., 1987]. Recent studies have established that dopamine is actively produced by lymphocytes [Bergquist et al., 1994; Musso et al., 1996] and modulates peripheral blood lymphocyte activity via dopamine D3 and D5 receptors [Ricci and Amenta,

() 1999 Wiley-Liss, Inc. 
1994; Barili et al., 1996] or via cellular uptake [Bergquist et al., 1994; Faraj et al., 1994]. The action of dopamine, well studied on target cells of the central nervous system, affects multiple intracellular pathways such as formation of cAMP, inositolphosphates and arachidonic acid, and alteration of the properties of ion channels [Mena et al., 1995; Lin et al., 1995; Van den Pol et al., 1996; Konradi et al., 1996]. Signals mediated by dopamine receptors D1 and D5, a D1like isoform, lead to increased cAMP levels and PKA activity, while D2-like receptors, such as the D2, D3, and D4 isoforms lead to a decrease of cAMP levels and inhibition of PKA activity. Depending on the cell type, pathways to the nucleus involve protein kinase $\mathrm{A}$ (PKA), protein kinase $\mathrm{C}(\mathrm{PKC})$ or tyrosine kinase proteins which regulate the activity of various transcription factors, such as CREB, AP-1, and the nuclear orphan receptor chicken ovalbumin upstream promoter transcription factor (COUPTF) [Miyajima et al., 1988; Wang et al., 1987, 1989], through transcriptional and post-transcriptional events [for review see Rogue and Malviya, 1994].

HIV-1 gene expression is controlled by viral and host cell transcription factors which interact with the long terminal repeat (LTR) region. Transcriptional activity of the HIV-1 promoter is mediated by the adjacent $\mathrm{Sp} 1$ and the $\kappa \mathrm{B}$ regulatory sequences of the LTR through the action of the transcription factors Sp1 and NF$\kappa B$. In addition, various modulatory sequences contribute to the regulation of the HIV-1 genome [for review see Kingsman and Kingsman, 1996]. Recently, a recognition sequence for members of the CREB/ATF family was identified between the lymphoid enhancer binding factor 1 (LEF-1) and the NF-кB sites [Krebs et al., 1997]. A downstream element was also identified as a novel TRE-like cAMP responsive element capable of binding AP- 1 and CREB/ATF within the untranslated leader region [Rabbi et al., 1997]. Dopamine has been described to activate COUP-TF by a phosphorylation-mediated event [Power et al., 1991]. We have reported that in neuronal cells, dopamine acts in concert with COUP-TF to stimulate HIV-1 gene transcription, via the proximal region of the LTR [Sawaya et al., 1996].

The effects of dopamine on HIV-1 gene expression in $\mathrm{T}$ cells have not yet been examined. Moreover, since the activities of the transcription factors CREB and COUP-TF are both modulated by dopamine and cAMP, and that dopamine binding modulates the cAMP pathway, it was of interest to examine how these factors regulate HIV-1 gene expression in the presence of dopamine and cAMP.

\section{MATERIALS AND METHODS Materials}

Cell culture media and all reagents, unless specified, were obtained from Sigma. $\left({ }^{14} \mathrm{C}\right)$ chloramphenicol was obtained from NEN Life Science. The LTR -45/-19, -25/-1, -5/+25, +20/+57, and $+57 /+86$ oligonucleotides were synthesized as two complementary single strand DNA by Eurogentech.

\section{Plasmid Constructs}

The LTR(LAI)-CAT, pRSV-COUP-TFI, and pRSV-COUPdel48 vectors were described previously [Sawaya et al., 1996; Rohr et al., 1997]. GC-WAP-CAT, GCmut-WAP-CAT (gift of Dr. B.E. Sawaya, Philadelphia) contain respectively the Wild-type and mutant (-79/-45) GCrich region from HIV-1 LTR [Taylor et al., 1992].

\section{Cell Culture, Transfections, and CAT Assays}

Jurkat $\mathrm{T}$ cells were grown in RPMI 1640 supplemented with $10 \%$ fetal calf serum, 10 $\mathrm{mM}$ HEPES, and penicillin/streptomycin (100 units/ml). Cells were transfected by the DEAEdextran method with 1 pmol of LTR-CAT reporter plasmid and $0.5 \mathrm{pmol}$ of expression vector: RSV-COUP-TF (gift of Dr. M.J. Tsai; Houston, TX), CMV-Sp1 (gift of Dr. R. Tjian, Berkeley, CA), RSV-CREB (gift of Dr. G. Schütz, Heidelberg, Germany), RSV-K-CREB (gift of Dr. F. Guillou, INRA, Tours, France). When indicated, cells were treated with dopamine $(100 \mu \mathrm{M})$, dibutyryl cAMP (dBcAMP; $0.5 \mathrm{mM})$, forskolin $(50 \mu \mathrm{M}), 24 \mathrm{~h}$ after transfection, and incubated for another $24 \mathrm{~h}$ before harvesting. Cell extracts were prepared $48 \mathrm{~h}$ after transfection. Each transfection was repeated at least four times with different plasmid preparations. Chloramphenicol acetyltransferase (CAT) assays were performed as described previously [Sawaya et al., 1996]. Acetylated and nonacetylated forms of chloramphenicol were extracted with ethyl acetate and separated by thin layer chromatography on silica gel. Percentage conversion of $\left({ }^{14} \mathrm{C}\right)$ chloramphenicol to its acetylated forms was quantified with a phosphorimager (Fuji). In cotransfections experiments, CAT 
activities were expressed relative to the value obtained with the corresponding LTR-CAT reporter vector, taken as 1 .

\section{Electrophoretic Mobility Shift Assays}

EMSAs were performed with nuclear proteins extracted from Jurkat cells as described previously [Sawaya et al., 1996]. Mixtures were incubated for 15 min at $4^{\circ} \mathrm{C}$ and protein-DNA complexes were analyzed by electrophoresis on a $6 \%$ polyacrylamide gel in $0.25 \times \mathrm{TBE}$. For supershift assays, antibodies directed against ATF-1/CREB (sc-270X; Santa Cruz Biotechnology) or normal rabbit serum, were mixed with nuclear proteins for $4 \mathrm{~h}$ at $4^{\circ} \mathrm{C}$ prior addition of the probe.

\section{Immunoprecipitation}

COUP-TF and CREB proteins were prepared by in vitro translation using the TNT T7 system (Promega). One-fifth of in vitro translated proteins was incubated in $400 \mu \mathrm{l}$ of TNE $(50 \mathrm{mM}$ Tris $\mathrm{pH} 8.0,1 \%$ Nonidet, $2 \mathrm{mM}$ EDTA, and a mixture of protease inhibitors), mixed with protein A-agarose beads $(20 \mu \mathrm{l})$ and gently shaken for $1 \mathrm{~h}$ at $4^{\circ} \mathrm{C}$. The suspension was briefly centrifugated and the supernatant was mixed with $2 \mu$ lof antibodies directed against ATF-1/
CREB (sc-270X; Santa Cruz Biotechnology), COUP-TF (sc-6577X; Santa Cruz Biotechnology) or with $2 \mu$ of nonimmune serum. After overnight incubation at $4^{\circ} \mathrm{C}$, protein A-agarose $(20 \mu \mathrm{l})$ was added and mixed for $2 \mathrm{~h}$. After extensive washing of the beads with TNE, $15 \mu \mathrm{l}$ of the beads were subjected to SDS-PAGE.

\section{RESULTS}

\section{Dibutyryl cAMP, Forskolin, and Dopamine \\ Stimulate HIV-1 LTR-Driven Transcription in Jurkat Cells}

To examine the effects of cAMP and dopamine on HIV-1 gene transcription, Jurkat cells were transfected with a LTR(LAI)-CAT reporter vector, and subsequently treated for $24 \mathrm{~h}$ with either the cAMP analog dibutyryl cAMP (dBcAMP), forskolin, an activator of adenylate cyclase, or dopamine (Fig. 1). Analysis of chloramphenicol acetyl transferase (CAT) activity was performed $48 \mathrm{~h}$ after transfection. Results showed that dBcAMP, forskolin, and dopamine stimulate CAT activity 2.0-, 3.3-, and 3.1-fold, respectively. Interestingly, treatment with both dopamine and forskolin resulted in a 9.7-fold synergistic transcriptional increase.

To delineate the LTR sequences responsible for induced HIV-1 transcriptional activation,

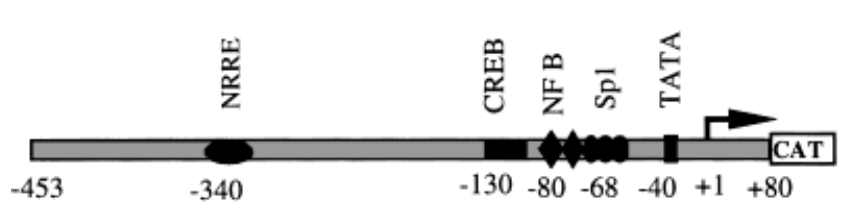

$-68$
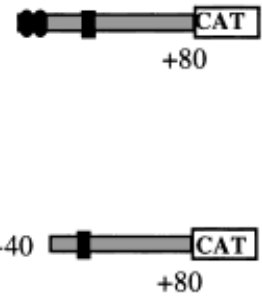

Relative CAT activity

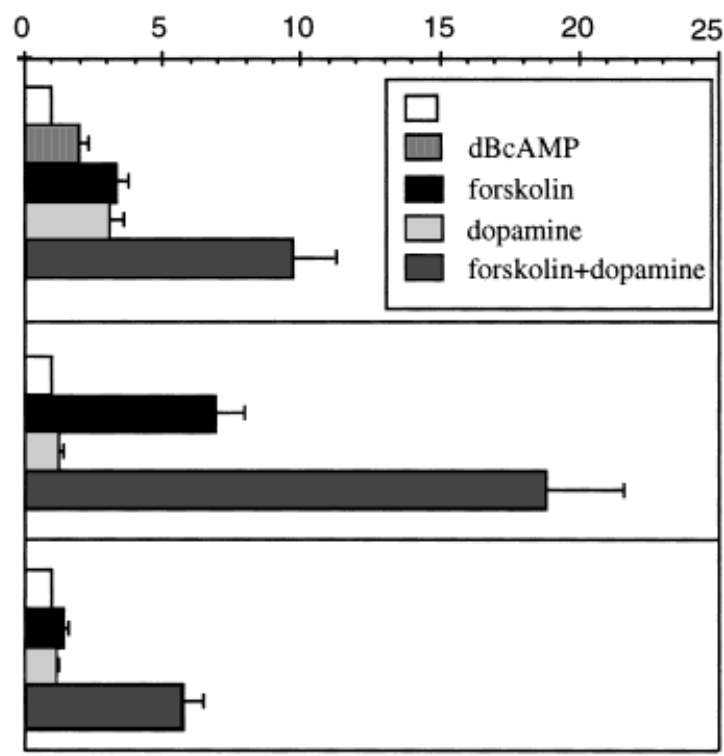

Fig. 1. Dibutyryl cAMP, forskolin and dopamine stimulate HIV-1 LTR-directed transcription in Jurkat cells. Jurkat cells were transfected with the HIV-1 LTR-CAT reporter vectors shown on the left panel and either left untreated or treated for $24 \mathrm{~h}$ with either dibutyryl cAMP $(0.5 \mathrm{mM})$, forskolin $(50 \mu \mathrm{M})$ or dopamine $(100 \mu \mathrm{M})$, as indicated. Cells extracts were prepared $48 \mathrm{~h}$ after transfection and CAT assays were performed. Error bars represent standard deviations from three experiments. 
cells were transfected with LTR-CAT vectors containing 5'-deleted LTR regions, and subsequently treated as above (Fig. 1). Forskolin alone stimulated the CAT activity of $-68 /+80$ LTR-CAT and -40/+80 LTR-CAT, 7.0- and 1.4fold, respectively. With the combination of forskolin and dopamine, CAT activity was stimulated 19- and 5.6 -fold, with -68/+80 LTR-CAT and $-40 /+80$ LTR-CAT, respectively. These results show that the transcriptional stimulation induced by forskolin and the combination of the two stimuli can be mediated through the $-68 \pm 80$ LTR region, containing only two Sp1 sites, and even, although at a lower extent, through the minimal -40/+80 LTR region. Dopamine alone was unable to affect transcription from the truncated regions, which indicates that the effect of dopamine is mediated by an element located upstream from position -68. Further experiments are needed to examine more precisely the nature of this element.

COUP-TF Mediates Forskolinand Dopamine-Induced HIV-1 Gene Transcription via the Proximal $-68 /+80$ LTR Region

The transcription factor COUP-TF belongs to the steroid/thyroid/retinoid receptor superfamily of transcription factors [Wang et al., 1989] and was shown to mediate dopamine responsiveness via the cAMP pathway [Power et al, 1991]. The presence of COUP-TF has been described in all tissues and in Jurkat cells [Miyajima et al., 1988; Cooney et al., 1991]. We have previously described that in neuronal cells, COUP-TF and dopamine stimulate HIV-1 gene transcription via the $-68 /+29$ region of the LTR [Sawaya et al., 1996]. We have also reported that COUP-TF acts as a transcriptional activator in microglial cells, via the proximal $-68 /+29$ LTR region [Rohr et al., 1997]. However, the effect of COUP-TF on HIV-1 gene transcription in lymphocytes has not been reported yet. When cotransfection experiments were performed with LTR-CAT and the COUP-TF expression vector (Fig. 2A,B, lane 2), CAT activity was stimulated 3.9-fold, which indicates that COUP-TF also acts as an activator of HIV-1 gene transcription in Jurkat cells. When cells were subsequently treated with forskolin or dopamine, CAT activities were further increased 10.5- and 13.3- fold (Fig. 2, lane 2), which confirms that COUP-TF also mediates cAMP and dopamine responsiveness.
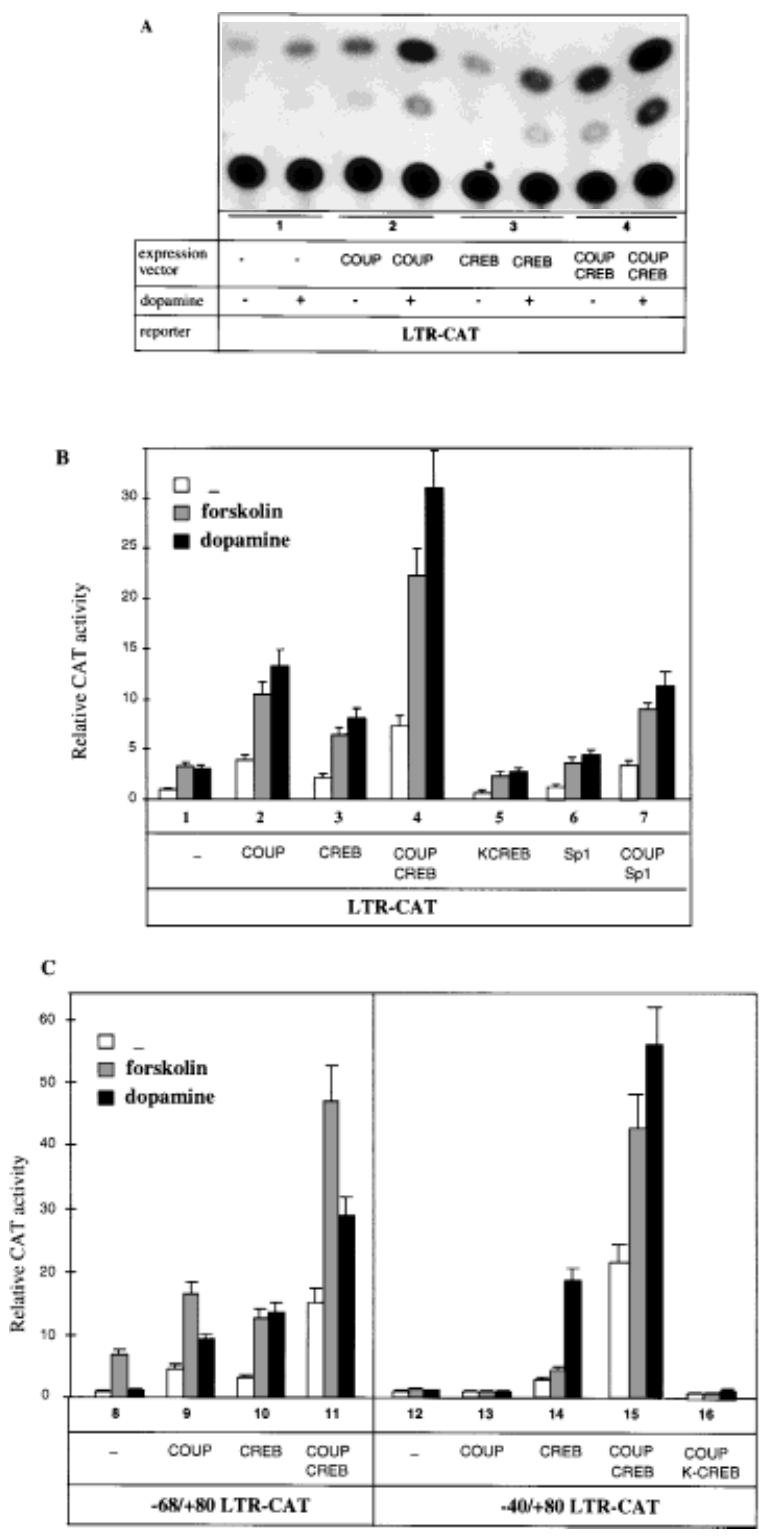

Fig. 2. Effect of the transcription factors CREB and COUP-TF on HIV-1 gene transcription in the presence of forskolin or dopamine. A: A representative CAT assay, performed after transient expression experiments in Jurkat cells, cotransfected with LTR-CAT and the indicated expression vector, in the absence or presence of dopamine treatment. B,C: Histograms represent CAT activities expressed relative to the value obtained with the corresponding LTR-CAT reporter vector, taken as 1, LTR-CAT, -68/+80 LTR-CAT, or -40/+80 LTR-CAT (1 pmol) and vectors expressing either CREB, K-CREB, COUP-TF, or Sp1 (0.5 pmol). Cells were treated or not with forskolin $(50 \mu \mathrm{M})$ or dopamine $(100 \mu \mathrm{M})$ for $24 \mathrm{~h}$. Values correspond to an average of at least three independent experiments done in duplicate.

With the truncated -68/+80 LTR-CAT vector, overexpression of COUP-TF led to a 4.6 -fold CAT stimulation (Fig. 2C, lane 9). In the presence of forskolin and dopamine, overexpression of COUP-TF led to a 16.4- and 9.3-fold CAT 
stimulation, respectively. In contrast, with -40/+80 LTR-CAT, overexpression of COUP-TF did not result in any change of CAT activity (Fig. 2C, lane 13). These findings clearly indicate that COUP-TF mediates transactivation, as well as forskolin and dopamine responsiveness, via the $-68 /+80$ sequence of the LTR.

\section{CREB Mediates Forskolin- and \\ Dopamine-Induced HIV-1 Gene Transcription via the Basal $-40 /+80$ LTR Region}

It is well known that the action of cAMP is mediated through the family of the transcription factors CREB/ATF [Lalli and SassoneCorsi, 1994]. While the action of dopamine in cells of the central nervous system has been shown to be essentially mediated by cAMP [Rogue and Malviya, 1994], in lymphocytes, these studies remain to be done. We therefore examined which region of the HIV-1 LTR was responsible for mediating the action of the transcription factor CREB, in the absence or presence of forskolin and dopamine. When Jurkat cells were cotransfected with the full length LTR-CAT vector and the CREB expression vector, the basal CAT level was increased 2.2-fold (Fig. 2A,B, lane 3). Treatment of cells with forskolin or dopamine led to a 6.4- and 8.1-fold stimulation of CAT activity, respectively (Fig. 2, lane 3). This result indicates that CREB is able to mediate both forskolin and dopamine responsiveness.

With the truncated -68/+80 LTR-CAT vector, containing only two Sp1 sites and the TATA box, overexpression of CREB led to a 3.2-fold stimulation (Fig.2C, lane 10). This finding indicates that the previously described CREB/ATF binding site located next to the NF-кB element [Krebs et al., 1997] is not essential for CREBmediated transactivation. In the presence of forskolin or dopamine, CAT stimulation was further increased 12.7 and 13.7-fold, respectively (lane 10).

Interestingly, with the vector containing the $-40 /+80$ basal LTR sequences, overexpression of CREB was still able to stimulate transcription 2.8-fold in the absence of any stimulus, and 4.5- and 18.6- fold, in the presence of forskolin or dopamine, respectively (Fig. 2C, lane 14). These results clearly demonstrate that CREB is able to mediate forskolin- and dopamineinduced transcriptional stimulation through the minimal $-40 /+80$ LTR sequences. This region does not contain any CRE or CRE-like se- quence. However, interactions of CREB with the basal transcription factors TFIIB, TFIID, and the adaptor CBP have already been demonstrated [Ferreri et al., 1994; Xing et al., 1995; Chrivia et al., 1993].

When similar cotransfection experiments were performed with LTR-CAT and the K-CREB mutant vector, containing a mutation which prevents DNA binding and functions like a dominant negative mutant [Walton et al., 1992], the basal and dopamine-induced CAT activities were not significantly affected (Fig. 2B, lane 5). This result confirms the importance of wildtype CREB in mediating cAMP and dopamine signals, and suggests that the DNA binding region of CREB is required for transactivation.

\section{CREB and COUP-TF Synergistically Activate Forskolin- and Dopamine-Induced HIV-1 Transcriptional Stimulation}

Since CREB and COUP-TF are able to mediate HIV-1 LTR-driven transcriptional stimulation, as well as dopamine and forskolin responsiveness, it was of interest to examine how HIV-1 gene transcription is modulated by the combination of both factors. With full length LTR-CAT, overexpression of both CREB and COUP-TF led to an additive CAT activity; when cells were further treated with forskolin or dopamine, CAT activity was enhanced 22- and 31-fold, respectively (Fig. 2A,B, lane 4). As presented above, forskolin and dopamine led to a 6.4- and 8.1-fold increase in the presence of CREB alone (Fig. 2, lane 3) and to a 10.5- and 13.3-fold increase in the presence of COUP-TF alone (Fig. 2, lane 2). These results indicate that in the presence of both factors, each stimulus leads to a synergistic transcriptional increase, compared with the effect obtained with either CREB or COUP-TF alone.

As could be expected, with -68/+80 LTR-CAT, the combined action of CREB and COUP-TF also led to a synergistic enhancement of transcriptional activity, in uninduced and forskolinor dopamine-induced conditions, since CAT activity was increased 15.2-, 47-, and 29- fold, respectively (Fig. 2C, lane 11).

It was interesting to test the effects of the combination of the two factors on the $-40 /+80$ LTR region, since only CREB, and not COUP$\mathrm{TF}$, was able to transactivate this region. With $-40 /+80$ LTR-CAT, as decribed above, overexpression of CREB led to a 2.8- and 4.5- fold increase, in the absence or presence of forskolin 
(Fig. 2, lane 14). Overexpression of both COUP-TF and CREB led to a synergistic 20and 47-fold increase in CAT activity, in the absence or presence of forskolin (Fig. 2, lane 15). These results reveal a functional synergy between CREB and COUP-TF, and suggest that these two proteins do interact, directly or indirectly.

We have previously reported that COUP-TF and $\mathrm{Sp} 1$ interact, and synergistically activate LTR-driven transcription in microglial cells [Rohr et al., 1997]. It was therefore interesting to examine how COUP-TF and Sp1 regulate HIV-1 gene transcription in Jurkat cells. Surprisingly, overexpression of $\mathrm{Sp} 1$ alone did not lead to a transcriptional increase, which may be explained by a high level of endogeneous Sp1 protein (Fig. 2B, lane 6). Moreover, the combination of Sp1 and COUP-TF was unable to change the level of expression obtained with each factor alone (Fig. 2, lanes 2, 6, 7). Dopamine treatment led to a 11-fold stimulation, similar to the 13-fold stimulation observed with COUP-TF alone (Fig. 2, lane 7). These findings reveal that in Jurkat cells, in contrast with microglial cells, COUP-TF does not synergize with Sp1, which stresses the importance of cell-type specific mechanisms which operate in the nucleus and regulate LTR-driven transcription.

\section{COUP-TF and CREB Do Not Interact Directly}

To test whether COUP-TF and CREB interact directly, co-immunoprecipitation experiments were performed with in vitro translated ${ }^{35}$ S-labeled COUP-TF and CREB proteins (Fig. 3 ). Results showed that anti-ATF-1/CREB antibodies were unable to coimmunoprecipitate the COUP-TF protein (Fig. 3, lane 3); vice-versa, anti-COUP-TF antibodies were unable to coimmunoprecipitate the CREB protein (Fig. 3, lane 6). As a control, anti-ATF1/CREB antibodies and anti-COUP-TF antibodies were able to immunoprecipitate CREB (Fig. 3, lane 1) and COUP-TF (Fig. 3, lane 4), respectively. These results indicate that COUP-TF and CREB do not interact directly in vitro. Moreover, coimmunoprecipitation experiments performed with extracts from Jurkat cells confirmed that anti-CREB antibodies were unable to coimmunoprecipitate the COUP-TF protein; vice-versa, anti-COUP-TF antibodies were unable to coimmunoprecipitate the CREB protein (results not shown), further indicating that COUP-TF and CREB do not interact directly.

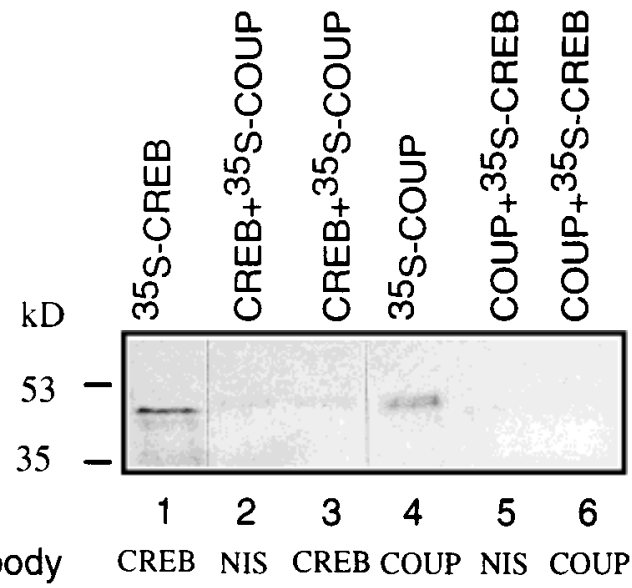

Fig. 3. CREB and COUP-TF do not interact directly in vitro. ${ }^{35}$ S-labeled COUP-TF was produced by in vitro translation using the TNT T7 kit (Promega) and incubated with in vitro translated unlabeled CREB, followed by immunoprecipitation with either non-immune serum (NIS, lane 2) or anti-ATF-1/CREB antibodies (lane 3). ${ }^{35}$ S-labeled CREB was translated in vitro, incubated with in vitro translated unlabeled COUP-TF, and immunoprecipitated with either non-immune serum (lane 5) or anti-COUP-TF antibodies (lane 6). As a control, ${ }^{35}$ S-CREB and 35S-COUP-TF were immunoprecipitated by their respective antibodies, antiATF-1/CREB (lane 1) and anti-COUP-TF (lane 4).

\section{Domains of COUP-TF and CREB Involved in the Transcriptional Synergy}

To investigate which region of CREB was required for the observed transcriptional synergy, -40/+80 LTR-CAT was cotransfected with COUP-TF and the mutant K-CREB. As seen in Fig. 2C (lane 16), K-CREB was unable to synergize with COUP-TF, in contrast with Wild-type CREB (Fig. 2C, lane 15). This result suggests that the DNA binding domain of CREB is required for the transcriptional synergistic effect with COUP-TF.

To examine which region of COUP-TF was involved in the synergistic effect, transfection assays were carried out with $-68 /+80$ LTR-CAT and either pRSV-COUP-TFI containing the full length COUP-TF or pRSV-COUPdel48, containing only the N-terminal region of COUP-TF. In the presence of overexpressed CREB, the full length and the truncated COUP-TF stimulated the CAT activity 11-and 14-fold, respectively. As a control, overexpression of CREB alone, led to a 3 -fold stimulation (results not shown). This result shows that the $\mathrm{N}$-terminal part of COUP$\mathrm{TF}$, containing the DNA binding domain, is sufficient for the transcriptional synergy with CREB. 


\section{CREB Stimulates HIV-1 Transcription Without Direct Interactions With the $-45 /+80$ LTR Region}

Since our results showed that CREB stimulates HIV-1 gene transcription via the basal $-40 /+80$ LTR region, it was interesting to examine whether CREB was directly binding to this region. Supershift experiments were performed with nuclear extracts from Jurkat cells and a series of oligonucleotide probes corresponding to the -45 to +85 LTR region, in the presence of either ATF-1/CREB antibodies or non immune serum (NIS). As seen on Figure 4, the DNA/ protein complexes formed with the various LTR probes were not altered in the presence of NIS or anti ATF-1/CREB. As a control, the specific DNA-protein complexes C2, C3, and C4 formed with the CRE probe, corresponding to the CREB binding consensus sequence [Sassone-Corsi et al., 1988] were supershifted in the presence of anti-ATF-1/CREB antibodies. These results clearly indicate that the CREB protein present in Jurkat nuclear extracts is unable to directly bind to the DNA sequences of the $-45 /+80$ LTR region. However, interactions of CREB with the basal transcription factors TFIIB, TFIID, and the adaptor CBP have already been reported

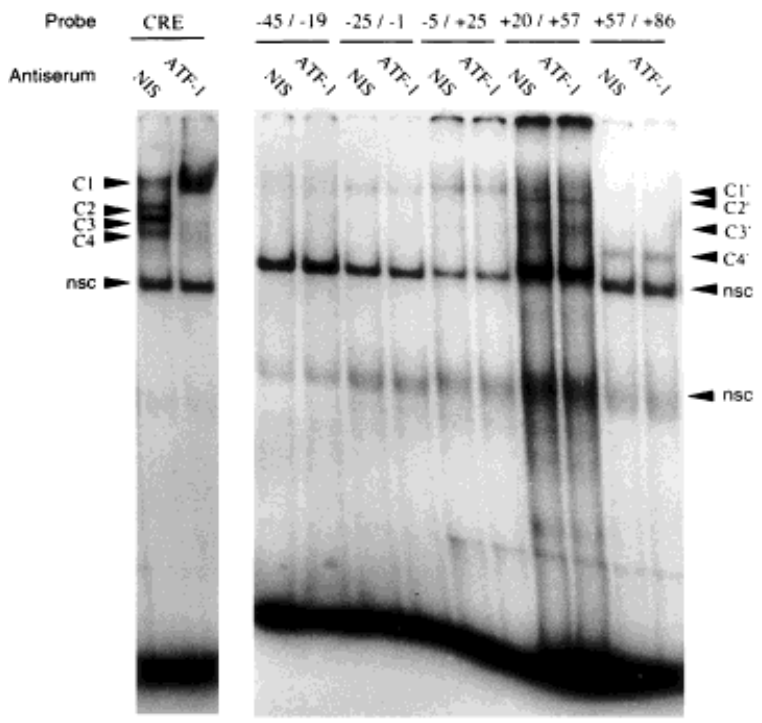

Fig. 4. CREB does not directly bind to the $-45 /+80$ region of the HIV-1 LTR. Supershift experiments were performed with nuclear extracts $(5 \mu \mathrm{g})$ from Jurkat cells, incubated in the presence of either non immune serum (lanes NIS) or anti-ATF-1/ CREB (lanes ATF-1). After $4 \mathrm{~h}$ at $4^{\circ} \mathrm{C}$, gel retardation assays were performed with $1 \mathrm{ng}$ of ${ }^{32} \mathrm{P}$ end-labeled $-45 /-19,-25 /-1,-5 /+25$, $+20 /+57,+57 /+86$ oligonucleotide probes corresponding to the LTR region, and the control CRE probe. The specific complexes $\left(\mathrm{C} 1-\mathrm{C} 4\right.$ and $\left.\mathrm{C}^{\prime}-\mathrm{C}^{\prime}\right)$ and the non-specific complexes (nsc) are indicated.
[Ferreri et al., 1994; Xing et al., 1995; Chrivia et al., 1993].

\section{Sp1 Sites are Sufficient for Forskolin- and Dopamine-Induced Synergistic Transcriptional Activation}

To precisely examine the role of the $\mathrm{Sp} 1$ sites present within the $-68 /+80$ LTR region in mediating the action of COUP-TF, either alone or in the presence of CREB, we used the GC-WAPCAT construct in which the -79/-45 Sp1 sequence of the HIV-1 LTR is inserted in a heterologous background. The Sp1 sites are located in front of the whey acidic protein (WAP) promoter containing a CAAT and a TATA box [Taylor et al., 1992]. As shown in Figure 5, transcription directed by the GC-WAP promoter was stimulated 28- and 4-fold, in the presence of overexpressed COUP-TF or CREB, respectively. A synergisitic 37-fold stimulation was observed with the combined action of COUP-TF and CREB. When cells were treated with dopamine, COUP-TF and CREB alone mediated a 44- and a 17-fold stimulation, respectively; the combination of both proteins led to a synergistic 90-fold stimulation. When cells were treated with forskolin, COUP-TF alone mediated a 76-

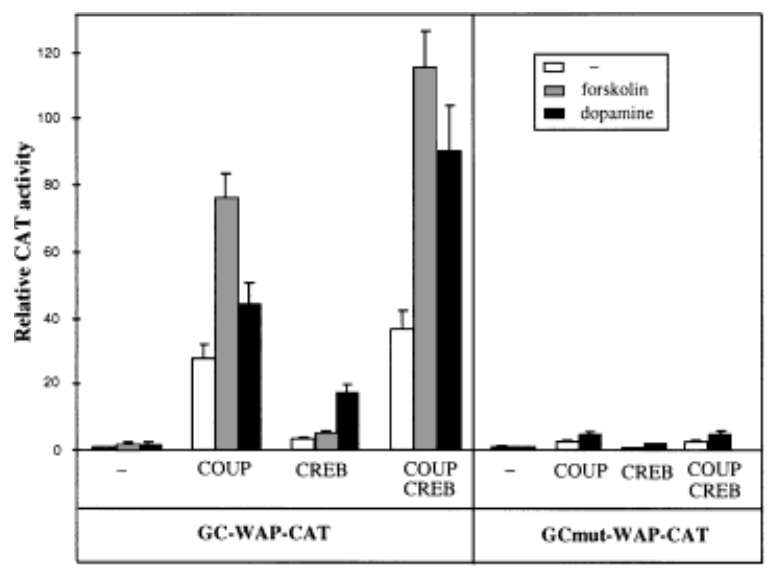

Fig. 5. Sp1 sites are sufficient for forskolin- or dopamineinduced synergistic transcriptional stimulation mediated by COUP-TF and CREB. The GC-WAP-CAT and GCmut-WAP-CAT vectors contain respectively the -45/-79 GC-rich wild-type and mutant sequence from the HIV-1 LTR, inserted upstream of the promoter of the whey acidic protein (WAP) and the CAT gene [Taylor et al., 1992]. GC-WAP-CAT or GCmut-WAP-CAT (1 pmol) were cotransfected in Jurkat cells with vectors expressing COUP-TF or CREB $(0.5 \mathrm{pmol})$ as indicated. Cells were treated or not with forskolin $(50 \mu \mathrm{M})$ or dopamine $(100 \mu \mathrm{M})$ for $24 \mathrm{~h}$. Cell extracts were prepared $48 \mathrm{~h}$ after transfection and CAT activities were determined. Values correspond to an average of at least three independent experiments. 
fold stimulation, CREB alone mediated a 4-fold stimulation, the combination of both proteins led to a synergistic 115 -fold stimulation. As a control, we transfected the GCmut-WAP-CAT vector, containing mutations within the Sp1 sites: with this vector, overexpression of COUP-TF or CREB was unable to significantly stimulate CAT activity. However, in this promoter background, the TATA region appeared unable to mediate CREB responsiveness. This contradiction revealed a promoter-specific action of CREB, which may be explained by the recruitment of different cofactors of CREB to the minimal LTR promoter or the formation of a LTR-specific transcriptional complex, compared with the GC-WAP promoter. Together, these results demonstrate that $\mathrm{Sp} 1$ sites, whether in the LTR or in a heterologous background, are sufficient to mediate both COUP-TF and CREB transcriptional activation, as well as the synergistic uninduced or stimuli-induced effects observed with both proteins.

\section{DISCUSSION}

In this report we have investigated the molecular mechanisms by which cAMP and dopamine regulate HIV-1 gene transcription in Jurkat $\mathrm{T}$ cells. In accordance with previous studies, our data indicate that elevation of cAMP concentrations by dBcAMP or forskolin treatment of Jurkat cells increases the transcriptional activity of the HIV-1 LTR. Our novel findings reveal that HIV-1 gene transcription in T cells is also modulated by dopamine. Interestingly, our results reveal a 15-fold synergistic increase of HIV-1 transcription following simultaneous stimulation with cAMP and dopamine. Our preliminary data also show that dopamine, similar to forskolin, enhances HIV-1 viral replication. Therefore, dopamine may play an important role in HIV-1 gene expression following activation of the cAMP pathway, itself activated upon infection with HIV-1.

Our transient expression data show that these two stimuli are able to mediate their signals through two distinct transcription factors, the CREB protein, well known to integrate cAMP signals at the gene activation level [Lalli and Sassone-Corsi, 1994] and the nuclear receptor COUP-TF, known to be activated by the dopamine signaling pathway via cAMP [Power et al., 1991].

We show here that the CREB protein stimulates HIV-1 gene transcription, and mediates forskolin as well as dopamine responsiveness in Jurkat cells. Moreover our results demonstrate that the action of CREB is mediated through the minimal $-40 /+80$ LTR region. Since supershift experiments show no direct DNA binding of CREB to this region, these novel findings suggest that CREB acts on the HIV-1 LTR by interacting with the general transcription factors TFIID or TFIIB, as previously reported [Ferreri et al., 1994; Xing et al., 1995] or with the adaptor CBP (CREB-binding protein) known to interact with the general transcription machinery [Nordheim et al., 1994]. This result also indicates that the CREB binding site identified between the LEF-1 and NF- $\mathrm{KB}$ sites [Krebs et al., 1997] is not an essential target for CREB-mediated activation. Proteins of the CREB/ATF family play an essential role in neuroendocrine processes, in spermatogenesis and are involved in circadian rhythms [Lalli and Sassone-Corsi, 1994]. The importance of CREB in dopamine signaling has been well established in the central nervous system, where phosphorylation of CREB is a critical event coupling dopamine stimulation to gene regulation [Cole et al., 1994; Liu et al., 1996]. Here our data establish the role and describe the target site of CREB for CAMP and dopamine signaling and HIV-1 gene regulation in cells of the immune system.

Our data further reveal that the nuclear receptor COUP-TF is also able to function as an activator of HIV-1 LTR-driven transcription in Jurkat cells, similar to its action in glial and microglial cells [Sawaya et al., 1996; Rohr et al., 1997]. Interestingly, the $-68 /+80$ LTR region, containing only two Sp1 binding sites, is sufficient for COUP-TF-mediated stimulation. The importance of these sites was confirmed by using the heterologous GC-WAP promoter, containing three $\mathrm{Sp} 1$ sites, also able to mediate the action of COUP-TF as well as responsiveness to forskolin and dopamine, in contrast with the GCmut-WAP promoter. This result is similar to the one previously described in microglial cells, where COUP-TF was shown to synergistically stimulate LTR-driven transcription by direct interaction with the Sp1 protein [Rohr et al., 1997]. In contrast, in glial TC-620 cells, COUP-TF stimulates transcription by direct interaction with the downstream-located nuclear receptor responsive element (NRRE) [Sawaya et al., 1996]. These data confirm that COUP-TF, acting through different mecha- 
nisms, functions as a multipotent transcriptional activator of HIV-1 gene expression, not only in brain cells, but also in cells of the immune system. Moreover they stress the importance of COUP-TF, involved in the response to cAMP and dopamine signals.

In addition, our data reveal the existence of a functional cooperation between the two transcription factors CREB and COUP-TF, which synergistically stimulate HIV-1 gene transcription. It appeared that the DNA binding domains of both proteins is involved in this transcriptional synergistic effect. Interestingly, overexpression of both proteins leads to a dramatic synergistic transcriptional activation upon forskolin or dopamine treatment. As a result, the combination of both proteins and both stimuli leads to a dramatic increase of LTR-driven transcription. Functional interactions between COUP-TF and CREB were also reported in the regulation of transferrin gene expression in Sertoli cells, where COUP-TF functions as a repressor of CREB-mediated stimulation [Suire et al., 1996]. These data suggest that depending on the promoter and the cell context, interactions between COUP-TF and CREB lead to synergistic or antagonistic effects. Our co-immunoprecipitation experiments indicate however that these two proteins do not interact directly.

Taken together, these studies reveal the importance of the transcription factors CREB and COUP-TF and of the minimal $-68 /+80$ HIV-1 LTR region, which integrates various activation signals transmitted to the viral LTR. However, further experiments are clearly needed to clarify the precise molecular mechanisms underlying dopamine signaling in lymphocytes, especially in primary cells, and the complex dopamine-mediated transactivation of the HIV-1 LTR.

\section{ACKNOWLEDGMENTS}

We thank N. Israël for providing the vectors containing the LTR(LAI). We are grateful to M.J. Tsai, G. Shütz, R. Tjian, B.E. Sawaya for providing the COUP-TF, CREB, Sp1, and GC-WAP-CAT expression vectors, respectively. We thank D. Lecestre for excellent technical assistance. This work was supported by the Institut National de la Santé et de la Recherche Médicale, the Agence Nationale des Recherches sur le SIDA, the Fondation pour la Recherche Medicale (FRM), the association "Le Cercle
d'Emeraude," la région Alsace. O.R. received financial support from the association "Ensemble contre le SIDA" and FRM (Sidaction).

\section{REFERENCES}

Barili P, Bronzetti E, Felici L, Ferrante F, Ricci A, Zaccheo D, Amenta F. 1996. Age-dependent changes in the expression of dopamine receptor subtypes in human peripheral blood lymphocytes. J Neuroimmunol 71:45-50.

Bergquist J, Tarkowski A, Ekman R, Ewing A. 1994. Discovery of endogenous catecholamines in lymphocytes and evidence for catecholamine regulation of lymphocyte function via an autocrine loop. Proc Natl Acad Sci USA 91:12912-12916.

Chrivia JC, Kwok RP, Lamb N, Hagiwara M, Montminy MR, Goodman RH. 1993. Phosphorylated CREB binds specifically to the nuclear protein CBP. Nature 365:855859.

Cole DG, Kobierski LA, Konradi C, Hyman SE. 1994. 6-Hydroxydopamine lesions of rat substantia nigra up-regulate dopamine-induced phosphorylation of the cAMPresponse element-binding protein in striatal neurons. Proc Natl Acad Sci USA 91:9631-9635.

Cooney AJ, Tsai SY, O’Malley BW, Tsai MJ. 1991. Chicken ovalbumin upstream promoter transcription factor binds to a negative regulatory region in the human immunodeficiency virus type 1 long terminal repeat. J Virol 65:28532860.

Faraj BA, Olkowski ZL, Jackson RT. 1994. Active [ $\left.{ }^{3} \mathrm{H}\right]-$ dopamine uptake by human lymphocytes: Correlates with serotonin transporter activity. Pharmacology 48:320 327.

Ferreri K, Gill G, Montminy M. 1994. The cAMP-regulated transcription factor CREB interacts with a component of the TFIID complex. Proc Natl Acad Sci USA 91:12101213.

Hofmann B, Nishanian P, Nguyen T, Insixiengmay P, Fahey JL. 1993a. Human immunodeficiency virus proteins induce the inhibitory cAMP/protein kinase A pathway in normal lymphocytes. Proc Natl Acad Sci USA 90:66766680 .

Hofmann B, Nishanian P, Nguyen T, Liu M, Fahey JL. 1993b. Restoration of T-cell function in HIV infection by reduction of intracellular cAMP levels with adenosine analogues. Aids 7:659-664.

Kingsman SM, Kingsman AJ. 1996. The regulation of human immunodeficiency virus type-1 gene expression. Eur J Biochem 240:491-507.

Konradi C, Leveque JC, Hyman SE. 1996. Amphetamine and dopamine-induced immediate early gene expression in striatal neurons depends on postsynaptic NMDA receptors and calcium. J Neurosci 16:4231-4239.

Krebs FC, Goodenow MM, Wigdahl B. 1997. Neuroglial ATF/CREB factors interact with the human immunodeficiency virus type 1 long terminal repeat. J Neurovirol: S28-32.

Lalli E, Sassone CP. 1994. Signal transduction and gene regulation: the nuclear response to cAMP. J Biol Chem 269:17359-17362.

Lin CW, Miller TR, Witte DG, Bianchi BR, Stashko M, Manelli AM, Frail DE. 1995. Characterization of cloned human dopamine D1 receptor-mediated calcium release in 293 cells. Mol Pharmacol 47:131-139. 
Liu FC, Graybiel AM. 1996. Spatiotemporal dynamics of CREB phosphorylation: transient versus sustained phosphorylation in the developing striatum. Neuron 17:11331144.

Marasini B, Biondi ML, Agostini A. 1987. Circulating dopamine in Raynaud's phenomenon. Angiology 38:878-882.

Mena MA, Garcia de Yebenes MJ, Tabernero C, Casarejos MJ, Pardo B, Garcia de Yebenes J. 1995. Effects of calcium antagonists on the dopamine system. Clin Neuropharmacol 18:410-426.

Miyajima N, Kadowaki Y, Fukushige S, Shimizu S, Semba K, Yamanashi Y, Matsubara K, Toyoshima K, Yamamoto T. 1988. Identification of two novel members of erbA superfamily by molecular cloning: the gene products of the two are highly related to each other. Nucleic Acids Res 16:11057-11074.

Musso NR, Brenci S, Setti M, Indiveri F, Lotti G. 1996. Catecholamine content and in vitro catecholamine synthesis in peripheral human lymphocytes. J Clin Endocrinol Metab 81:3553-3557.

Nokta M, Pollard R. 1991. Human immunodeficiency virus infection: association with altered intracellular levels of cAMP and cGMP in MT-4 cells. Virology 181:211-217.

Nokta MA, Pollard RB. 1992. Human immunodeficiency virus replication: Modulation by cellular levels of cAMP. Aids Res Hum Retroviruses 8:1255-1261.

Nordheim A. 1994. Transcription factors. CREB takes CBP to tango [news; comment]. Nature 370:177-178.

Power RF, Lydon JP, Conneely OM, O'Malley BW. 1991. Dopamine activation of an orphan of the steroid receptor superfamily. Science 252:1546-1548.

Rabbi MF, Saifuddin M, Gu DS, Kagnoff MF, Roebuck KA. 1997. U5 region of the human immunodeficiency virus type 1 long terminal repeat contains TRE-like cAMPresponsive elements that bind both $\mathrm{AP}-1$ and CREB/ATF proteins. Virology 233:235-245.

Ricci A, Amenta F. 1994. Dopamine D5 receptors in human peripheral blood lymphocytes: a radioligand binding study. J Neuroimmunol 53:1-7.

Rogue P, Malviya AN. 1994. Regulation of signalling pathways to the nucleus by dopaminergic receptors. Cell Signal 6:725-733.

Rohr O, Aunis D, Schaeffer E. 1997. COUP-TF and Sp1 interact and cooperate in the transcriptional activation of the human immunodeficiency virus type 1 long terminal repeat in human microglial cells. J Biol Chem 272:31149 31155.

Sassone-Corsi P, Visvader J, Ferland L, Mellon PL, Verma IM. 1988. Induction of the proto-oncogene fos transcription through the adenylate cyclase pathway: characterization of a cAMP-responsive element. Genes Dev 2:15291538.

Sawaya BE, Rohr O, Aunis D, Schaeffer E. 1996. Chicken ovalbumin upstream promoter transcription factor, a transcriptional activator of HIV-1 gene expression in human brain cells. J Biol Chem 271:23572-23576.

Suire S, Maurel MC, Guillou F. 1996. Follitropin action on the transferrin gene in Sertoli cells is mediated by cAMPresponsive-element-binding-protein and antagonized by chicken ovalbumin-upstream-promoter-transcription factor. Eur J Biochem 239:52-60.

Taylor JP, Pomerantz R, Bagasra O, Chowdhury M, Rappaport J, Khalili K, Amini S. 1992. TAR-independent transactivation by Tat in cells derived from the CNS: a novel mechanism of HIV-1 gene regulation. Embo J 11:33953403.

Van den Pol AN, Cao V, Belousov AB. 1996. Dopamine enhancement and depression of glutamate-regulated calcium and electrical activity in hypothalamic neurons. J Neurophysiol 76:3934-3948.

Walton KM, Rehfuss RP, Chrivia JC, Lochner JE, Goodman RH. 1992. A dominant repressor of cyclic adenosine $3^{\prime}, 5^{\prime}$ monophosphate (cAMP)- regulated enhancer-binding protein activity inhibits the cAMP-mediated induction of the somatostatin promoter in vivo. Mol Endocrinol 6:647655.

Wang LH, Tsai SY, Cook RG, Beattie WG, Tsai MJ, O’Malley BW. 1989. COUP transcription factor is a member of the steroid receptor superfamily. Nature 340:163-166.

Wang LH, Tsai SY, Sagami I, Tsai MJ, O’Malley BW. 1987. Purification and characterization of chicken ovalbumin upstream promoter transcription factor from HeLa cells. J Biol Chem 262:16080-16086.

Xing L, Gopal VK, Quinn PG. 1995. cAMP response elementbinding protein (CREB) interacts with transcription factors IIB and IID. J Biol Chem 270:17488-17493. 